\title{
Scientific Visualization of the Results of a Numerical Experiment of the Nonlinear Dynamics of a Nanoscale Beam Structure*
}

\author{
Olga Saltykova [0000-0002-3880-6662] \\ Yuri Gagarin State Technical University, 77 Politehnicheskaya st., Saratov, Russia \\ olga_a_saltykova@mail.ru
}

\begin{abstract}
The paper presents the results of scientific visualization of the nonlinear dynamics of contact interaction of a nanoscale beam structure under the action of an external harmonic load. The beam structure consists of two beams obeying the kinematic hypotheses of Euler-Bernoulli and S.P. Timoshenko. The constructed mathematical model takes into account geometric and constructive nonlinearities. The size-dependent behavior of the structure is implemented on the basis of the modified moment theory of elasticity. The resulting system of partial differential equations is reduced to a system of ordinary differential equations by the second order finite difference method. The Cauchy problem is solved by the fourth order Runge-Kutta method. In this work, using the methods of scientific visualization of the results of applying the methods of nonlinear dynamics, the influence of the size-dependent parameter and external load on the vibrations of the beam structure is investigated. As methods for studying nonlinear dynamics, the work uses wavelet spectra based on the mother Morlet, Fourier power spectra, signals. The use of scientific visualization methods makes it possible to develop specific recommendations for the operating conditions of the beam structure. This, in turn, makes it possible to avoid unwanted vibration modes of beam nanostructures, which are widely used as sensitive elements of sensors of micro and nano electromechanical systems.
\end{abstract}

Keywords: Nanobeams, Nonlinear Dynamics, Wavelet Analysis, Timoshenko Model, Euler-Bernoulli Model, Modified Moment Theory of Elasticity.

Copyright (C) 2020 for this paper by its authors. Use permitted under Creative Commons License Attribution 4.0 International (CC BY 4.0).

* Supported by RFBR grant № 18-41-700001 r_a 


\section{Introduction}

Scientific visualization is one of the effective methods of analysis of data obtained as a result of numerical experiments, therefore, it is widely used in various kinds of research [1], [2]. The main objective of this work is the application of scientific visualization methods to study the nonlinear dynamics of a nanoscale beam structure.

Such beam structures are components of sensitive elements of various nanoelectromechanical systems and sensors and during the operation of such systems can be subjected to various external influences, including dynamic ones [3].

\section{Formulation of the problem}

The object of study is the beam structure, consisting of two nanobeams with a gap between them (Fig. 1). Beam 1 is described by the Euler-Bernoulli kinematic hypothesis, and beam 2 is described by the Timoshenko hypothesis, which takes into account the shear strain. $l$ - is a length, $2 h$ - is a thickness of beams. Both beams are geometrically non-linear according to the model of T. von Karman. A transverse alternating load (1) distributed over the surface acts on the beam 1, and the beam 2 only starts moving after contact with the beam 1 .

$$
q(x, t)=q_{0} \sin \left(\omega_{p} t\right),
$$

where $q(x, t)$ - is the alternating load; $\omega_{p}$-is the frequency; $q_{0}$ - is the amplitude; $t$ - it is time.

Contact interaction is described by the model of Cantor B.Ya. [4], that is, at each time step, the problem of contact of two bodies with an unknown contact boundary is solved. The equations of motion, boundary and initial conditions are obtained from the Hamilton-Ostrogradsky energy principle. Classical solid mechanics is not able to interpret and predict size-dependent behavior, due to the lack of a parameter that takes into account scale effects. Therefore, when obtaining the equations, we use the modified moment theory of elasticity [5], where the scale parameter of the material length is taken into account, taking into account the effect of higher-order moments $[6,7]$.

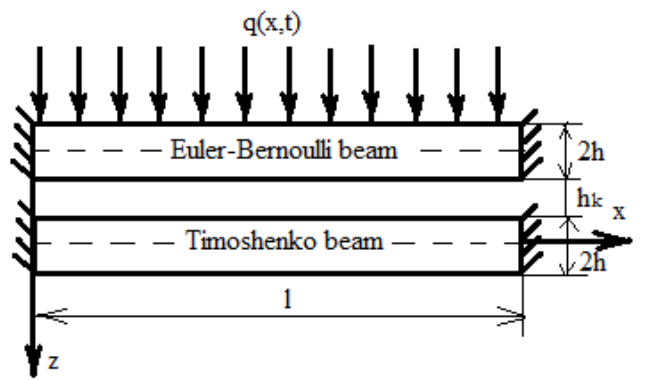

Fig. 1. Nanobeam structure 
The equations of motion of beams in a dimensionless form take the form:

$$
\begin{aligned}
& \int \frac{1}{\lambda^{2}}\left\{F_{2}\left(w_{i}, w_{i}\right)+F_{1}\left(u_{i}, w_{i}\right)+\left(-\frac{1}{12}+\frac{\mu^{2}}{2(1+v)}\right) \frac{\partial^{4} w_{1}}{\partial x^{4}}\right\}-\frac{\partial^{2} w_{1}}{\partial t^{2}}-\varepsilon_{1} \frac{\partial w_{1}}{\partial t}+ \\
& +(-1)^{i} K\left(w_{1}-w_{2}-h_{k}\right) \Psi+q(x, t)=0, \\
& \frac{\partial^{2} u_{1}}{\partial x^{2}}+F_{3}\left(w_{i}, w_{i}\right)-\frac{\partial^{2} u_{1}}{\partial t^{2}}=0 \\
& \left\{\frac{1}{3}\left(\frac{\partial^{2} w_{2}}{\partial x^{2}}+\frac{\partial \gamma_{x 2}}{\partial x}\right)+\frac{1}{\lambda^{2}}\left(L_{1}\left(w_{i}, u_{i}\right)+\frac{3}{2} L_{2}\left(w_{i}, w_{i}\right)+L_{3}\left(w_{i}, u_{i}\right)\right)+\right. \\
& +(-1)^{i} K\left(w_{1}-w_{2}-h_{k}\right) \Psi-\frac{\partial^{2} w_{2}}{\partial t^{2}}-\varepsilon_{1} \frac{\partial w_{2}}{\partial t}=0 ; \\
& \lambda^{2}\left(\frac{\partial^{2} u_{2}}{\partial x^{2}}+L_{4}\left(w_{i}, w_{i}\right)\right)-\frac{\partial^{2} u_{2}}{\partial t^{2}}=0 \\
& \lambda^{2} \frac{\partial^{2} \gamma_{x 2}}{\partial x^{2}}-\frac{6 \lambda^{4}}{1+v}\left(\frac{\partial w_{2}}{\partial x}+\gamma_{x 2}\right)+\frac{3 \lambda^{2}}{2(1+v)} \mu^{2}\left(\frac{\partial^{2} \gamma_{x 2}}{\partial x^{2}}-\frac{\partial^{3} w}{\partial x^{3}}\right)-\frac{\partial^{2} \gamma_{x 2}}{\partial t^{2}}=0 ; i=1,2, \\
& i=1,2 \text { - is the serial number of beams. } F_{1}\left(u_{i}, w_{i}\right)=\frac{\partial^{2} u_{1}}{\partial x^{2}} \frac{\partial w_{1}}{\partial x}+\frac{\partial u_{1}}{\partial x} \frac{\partial^{2} w_{1}}{\partial x^{2}} \text {, } \\
& F_{2}\left(w_{i}, w_{i}\right)=\frac{3}{2} \frac{\partial^{2} w_{1}}{\partial x^{2}}\left(\frac{\partial w_{1}}{\partial x}\right)^{2}, \quad F_{3}\left(w_{i}, w_{i}\right)=\frac{\partial^{2} w_{1}}{\partial x^{2}} \frac{\partial w_{1}}{\partial x}, \quad L_{1}\left(w_{i}, u_{i}\right)=\frac{\partial^{2} w_{i}}{\partial x^{2}} \frac{\partial u_{i}}{\partial x}, \\
& L_{2}\left(w_{i}, w_{i}\right)=\frac{\partial^{2} w_{i}}{\partial x^{2}}\left(\frac{\partial w_{i}}{\partial x}\right)^{2}, \quad L_{3}\left(w_{i}, u_{i}\right)=\frac{\partial w_{i}}{\partial x} \frac{\partial^{2} u_{i}}{\partial x^{2}}, L_{4}\left(w_{i}, w_{i}\right)=\frac{\partial w_{i}}{\partial x} \frac{\partial^{2} w_{i}}{\partial x^{2}} \text { are }
\end{aligned}
$$

nonlinear operators, $\gamma_{x i}$ is the transverse shear function, $w_{i}, u_{i}$ are the deflections and displacements of the beams, respectively, $h_{k}$ is the gap between the beams (see Fig. 1), $v=0.3$ is the Poisson's ratio, is the scale parameter of the material length, $\varepsilon_{1}$ is the dissipation coefficient, $\lambda=\frac{l}{2 h}$ is the geometrical parameter.

To model contact interaction, the theory of B.Ya.Cantor [4], according to which it is necessary to add a term to the equations of motion of structural elements $q_{k}=(-1)^{i} K_{1}\left(w_{1}-h_{k}-w_{2}\right) \Psi, K$ is the stiffness coefficient of the transverse compression of the structure in the contact zone. The function $\Psi$ is defined by the formula $\Psi=\frac{1}{2}\left[1+\operatorname{sign}\left(w_{1}-h_{k}-w_{2}\right)\right]$, (if $w_{1}>h_{k}+w_{2}$, then there is contact between the structural elements and $\Psi=1$; in the absence of $\operatorname{contact} \Psi=0$ ). 
To equations (2), boundary conditions in the case of rigid pinching (3), (5) and initial conditions (4), (6) for beams 1 and 2, respectively, should be added.

For beam 1:

$$
\begin{gathered}
w_{1}(0, t)=w_{1}(1, t)=u_{1}(0, t)=u_{1}(1, t)=\frac{\partial w_{1}(0, t)}{\partial x}=\frac{\partial w_{1}(1, t)}{\partial x}=0 . \\
w_{1}(x)_{\mid t=0}=0, u_{1}(x)_{\mid t=0}=0,{\frac{\partial w_{1}(x)}{\partial t}}_{\mid t=0}=0,{\left.\frac{\partial u_{1}(x)}{\partial t}\right|_{t=0}=0 .}=0
\end{gathered}
$$

For beam 2:

$$
\begin{gathered}
w_{2}(0, t)=w_{2}(1, t)=0 ; u_{2}(0, t)=u_{2}(1, t)=0 ; \\
\gamma_{x 2}(0, t)=\gamma_{x 2}(1, t)=0 ; \frac{\partial w_{2}(0, t)}{\partial x}=\frac{\partial w_{2}(1, t)}{\partial x}=0 ; \\
w_{2}(x, t)_{\mid t=0}=0, u_{2}(x, t)_{\mid t=0}=0, \gamma_{x 2}(x, t)_{\mid t=0}=0, \\
\left.\frac{\partial w_{2}(x, t)}{\partial t}\right|_{\mid t=0}=0,\left.\frac{\partial u_{2}(x, t)}{\partial t}\right|_{\mid t=0}=0,\left.\frac{\partial \gamma_{x 2}(x, t)}{\partial t}\right|_{\mid t=0}=0 .
\end{gathered}
$$

\section{Solution methods}

The resulting system of partial differential equations is reduced to an ordinary differential equation system by the second-order finite difference method. The obtained Cauchy problem is solved by the fourth-order Runge-Kutta method.

To study the nonlinear dynamics of the contact interaction of a nanoscale beam structure on the basis of nonlinear dynamics methods, we construct signals, plots, power spectra, wavelet spectra based on the Morlet mother wavelet, and phase portraits. The totality of the visual presentation of the results obtained by these methods will allow us to have an almost complete picture of the features of the nonlinear dynamics of the described mechanical structure, depending on the value of the size-dependent parameter $\mu$ and the amplitude of the external load $q_{0}$.

\section{$4 \quad$ Numerical experiment}

We are present the results of a numerical experiment with the following values of the control parameters: $\lambda=50 ; h_{k}=0.1 ; q_{0} \in\{1000 ; 5000\} ; \varepsilon=1 ; \mu \in\{0.1 ; 0.3 ; 0.5\}$.

Table 1 shows the signals of beams 1 (red) and 2 (blue) $w(t, 0.5), t \in(0 ; 20)$ for various values of the amplitude load $q_{0} \in\{1000 ; 5000\}$ and the size-dependent parameter $\mu \in\{0.1 ; 0.3 ; 0.5\}$.

Depending on the value of the size-dependent parameter $\mu$, the oscillation 
amplitude of the beam 1 changes, an increase in the parameter leads to a decrease in the amplitude of the oscillations. Visualization of the beam signals allows you to see the points in time at which the beam contacts. But, to determine the shape of the vibrations of the beams, it is necessary to analyze the plots of the deflections of the beams.

Table 1. Signals of the nanobeams for $q_{0} \in\{1000 ; 5000\} ; \varepsilon=1 ; \mu \in\{0.1 ; 0.3 ; 0.5\}$

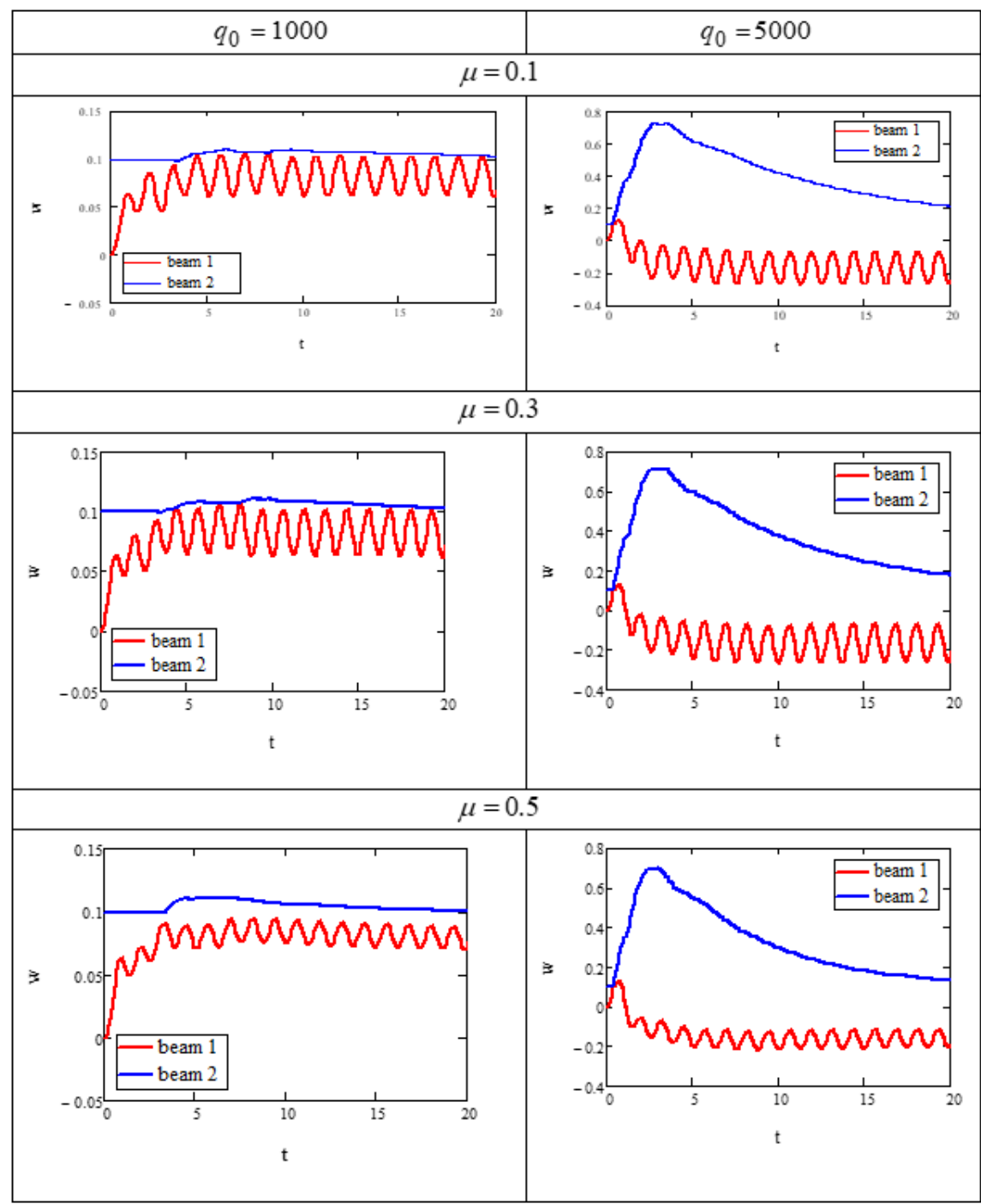

Table 2 shows the plots for $\mu=0.1, q_{0} \in\{1000 ; 5000\}$, at different points in time. Graphical visualization of deflection diagrams of a beam nanostructure allows obtaining information about the vibration mode at any moment of interest, which cannot be seen with only a numerical solution. 
Table 2. Plots of deflections of beams 1 and 2 .

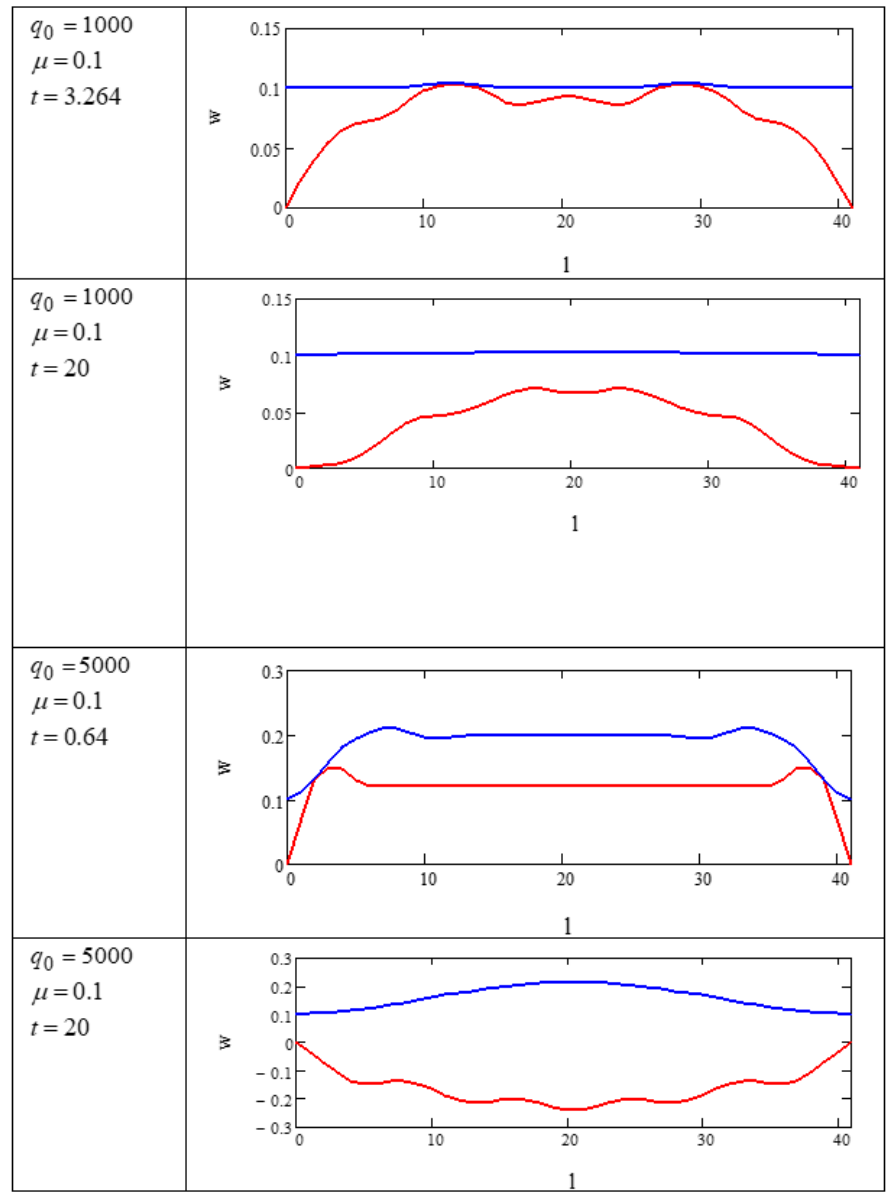

The diagrams at $\mu=0.1, q_{0}=1000$, are given for the moment of the first contact of the beams, at $t=3.264$. It was possible to determine the time of the first touch by visualizing the beams deflection diagrams in three-dimensional space $\Omega(x, z, t)$. As you can see, the first contact of the beams does not occur at the central point, but in quarters. The increase in load leads to the fact that the first contact of the beams occurs at $t=0.64$, and the appearance of the diagrams at the first contact changes. The visualization of vibration modes allows you to develop specific recommendations for the operating conditions of the studied mechanical structure.

To obtain the frequency characteristics of signals, we use the wavelet spectra based on the Morlet mother wavelet, as well as the Fourier power spectra. Wavelet analysis allows you to see the change in the frequency characteristics of the oscillatory process in time and is a kind of "microscope".

Tables 3 and 4 show wavelets $\omega_{p}(t)$ and Fourier power spectra for beams 1 and 2 at $q_{0}=1000$ (table 3 ) and at $q_{0}=5000$ (table 4). 
Scientific Visualization of the Results of a Numerical Experiment of the Nonlinear... 7

Table 3. Wavelet spectra at $q_{0}=1000 ; \mu \in\{0.1 ; 0.3 ; 0.5\}$

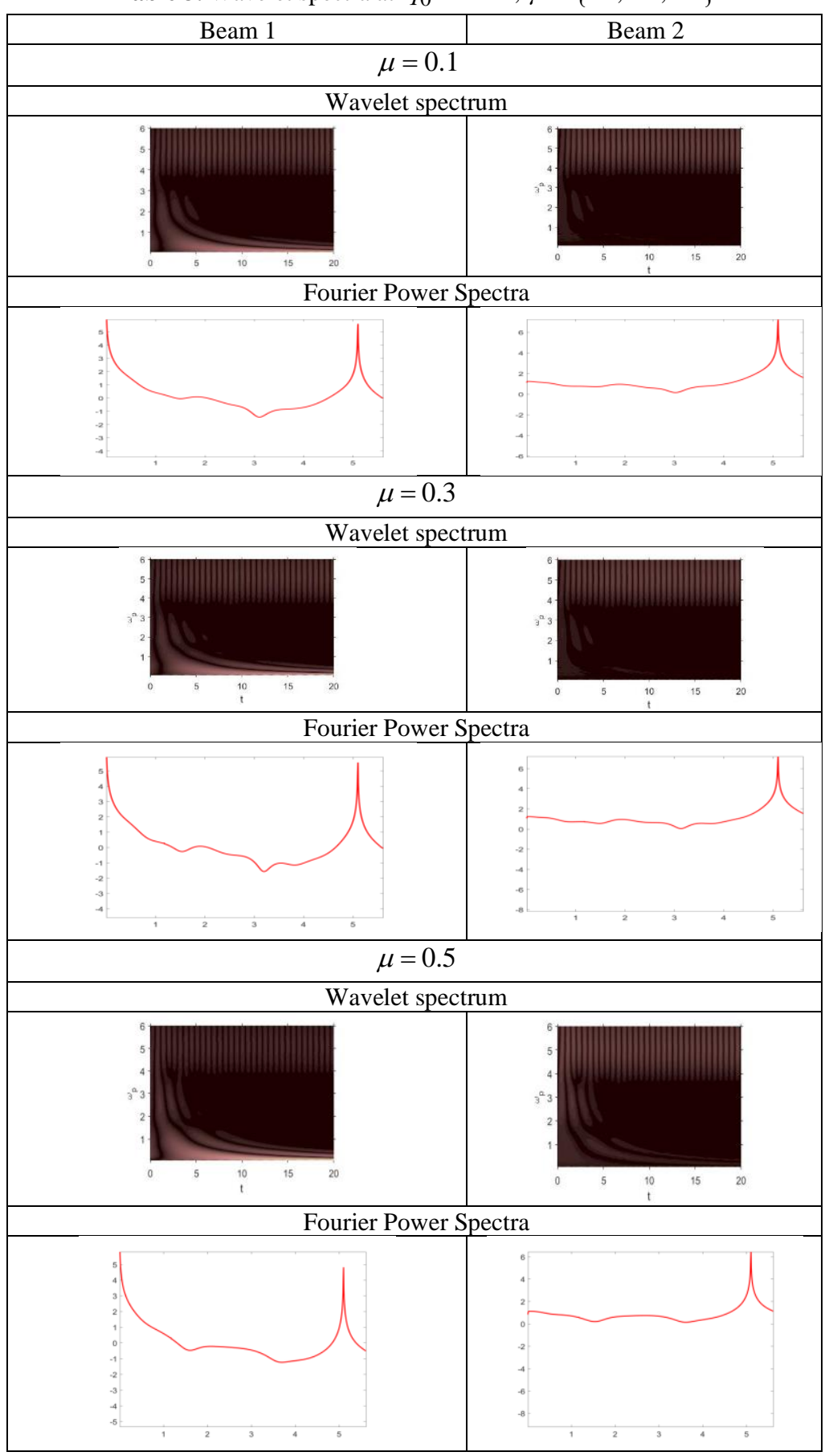


Table 4. Wavelet spectra at $q_{0}=5000 ; \mu \in\{0.1 ; 0.3 ; 0.5\}$

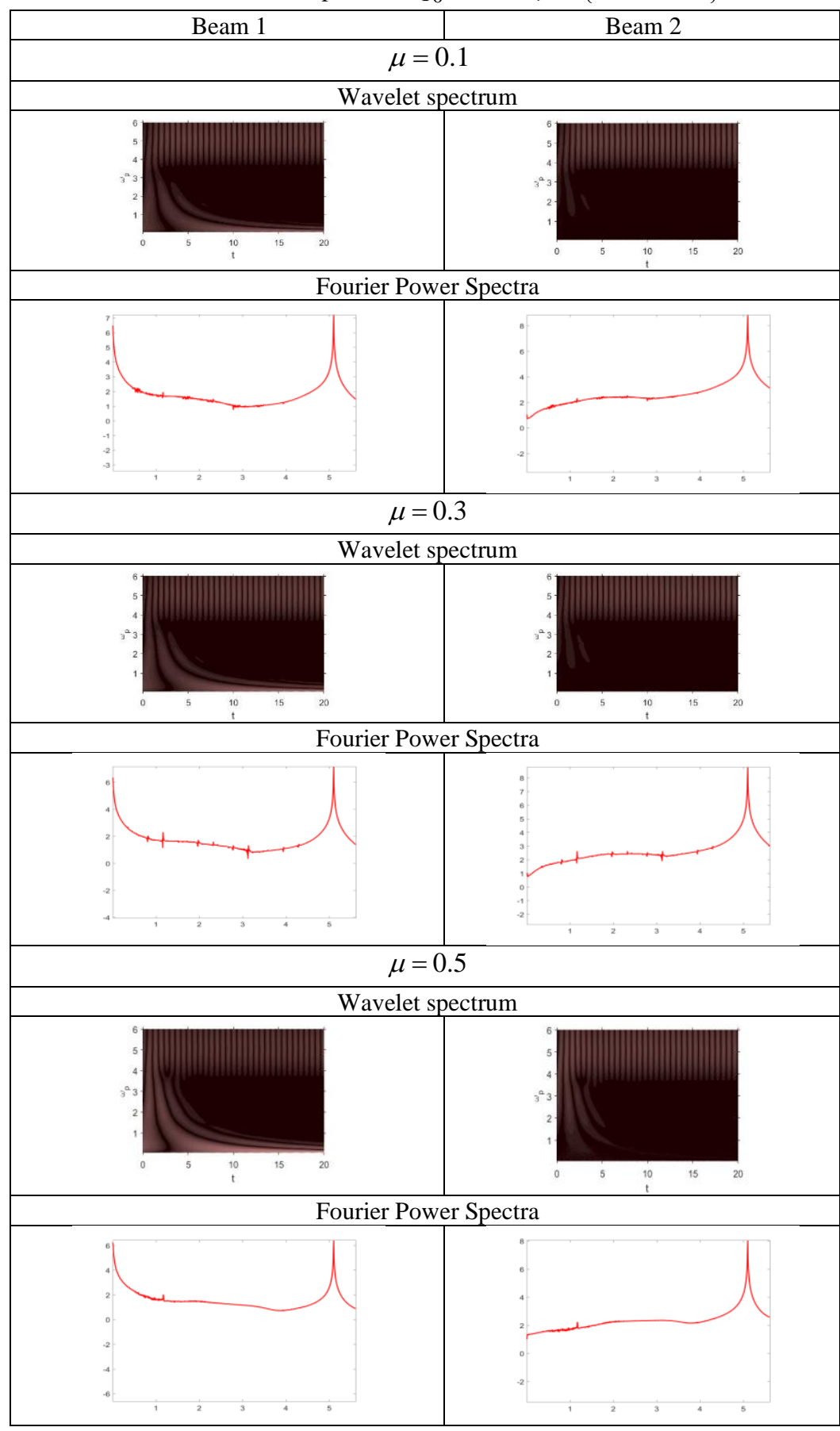


Wavelets are given for $t \in(0 ; 20)$, and the Fourier power spectra are built on the time interval $t \in(0 ; 1024)$. This is due to the fact that if the wavelet spectra are plotted over the entire time interval, the frequency changes characteristic of the initial time interval are not visible.

The wavelet spectra of beams 1 and 2 are distinguished by the presence of a lowfrequency component in the spectrum of beam $1 \mu=0.5$ when the wavelet spectra of beam 2 is generated, a low-frequency component occurs over the entire time interval. The Fourier power spectra do not differ in frequency components. The same frequencies can be seen on wavelet spectrum.

Table 4 shows the wavelet spectra and the power spectra of the Fourier beams at $q_{0}=5000$.

The wavelet spectra for reducible loads differ significantly for the same values of the size-dependent parameter, as well as the Fourier power spectra. An increase in the sizedependent parameter leads to a decrease in frequencies, i.e., to regularization of oscillations. This is due to a decrease in the amplitude of oscillations of the beams, which was mentioned above.

\section{Conclusions}

Scientific visualization of the nonlinear dynamics of the contact interaction of the beam structure allows you to have a detailed idea of the influence of control parameters on the nature of the vibrations of the beam structure, which makes it possible to develop specific recommendations on the operating conditions of the beam structure and avoid undesirable vibration modes, which can ultimately lead to the destruction of the beam structure.

\section{References}

1. Zakharova, A.A., Vekhter, E.V., Shklyar, A.V.: The applicability of visualization tools in the meta-design of an educational environment. European Journal of Contemporary Education, 8 (1), 43-51. (2019).

2. Brodlie, K. W., Carpenter, L. A., Earnshaw, R. A., Gallop, J. R., Hubbold, R. J., Mumford, A. M., ... \& Quarendon, P. (Eds.). Scientific visualization: techniques and applications. Springer Science \& Business Media. (2012).

3. Fu, Y., Zhang, J.: Electromechanical dynamic buckling phenomenon in symmetric electric fields actuated microbeams considering material damping. Acta Mech 212, 29-42, (2010)

4. Cantor, B.Ya.: Contact problems of the nonlinear theory of shells of revolution. In: Naukova dumka, Kiev (1991).

5. Toupin, R.A.: Elastic materials with couple-stresses. Arch. Ration. Mech. Anal. 11, 385414, (1962).

6. Simsek, M., Reddy, J.N.: Bending and vibration of functionally graded microbeams using a new higher order beam theory and the modified couple stress theory. International Journal of Engineering Science, 64, 34-53, (2013).

7. Krysko, V.A., Awrejcewicz, J., Papkova, I.V., Saltykova, O.A., Krysko, A.V.: Chaotic contact dynamics of two microbeams under various kinematic hypotheses. International Journal of Nonlinear Sciences and Numerical Simulation. 20(3-4), 373-386, (2019). 